\title{
Mapping of $2 D$ contact perturbations by electrons on a helium film
}

\author{
E. Teske ${ }^{1}$, P. Leiderer ${ }^{2}$, P. Wyder ${ }^{1}$, and V. Shikin ${ }^{3}$ \\ ${ }^{1}$ Grenoble High Magnetic Field Laboratory, Max-Plank Institut fur Festkorperforschung \\ Centre National de la Recherche Scientifique, B.P.166, F-38042, Grenoble, Cedex 9, France \\ ${ }^{2}$ Fakultät für Physik, Universität Konstanz, D-78434 Konstanz, Germany \\ ${ }^{3}$ Institute of Solid State Physics, 142432 Chernogolovka, Moscow District, Russia \\ E-mail: shikin@issp.ac.ru
}

\begin{abstract}
A promising way to investigate $2 D$ contact phenomena is proposed. This method is based on the idea of depositing surface state electrons (SSE) on a thin layer of liquid helium covering the surface of a solid sample containing a $2 D$ charge carrier system. The density of SSE adjusts to screen contact-induced perturbations of the electrostatic potential across the sample. As a result, the helium layer thickness varies due to the variation of the electrostatic pressure, thus providing a map. This map may be read off interferometrically by a technique already employed for the investigation of multi-electron dimples on helium. We have realized this mapping for a structured electrode as a test sample to demonstrate the resolution of the method.
\end{abstract}

PACS: $67.40 .-w, 67.70 .+n$

Contact phenomena are well known in $3 D$ metal and semiconductor physics $[1,2]$. We have in mind, e.g., the determination of work function for different $3 D$ metals, the solution of Shottky barrier problem and applications of this solution to different aspects of transistor physics, in particular the creation of heterostructures etc. In all these cases the perturbation of electron density near the boundary between the contacting metals or semiconductors is well localized within the so called Debye radius.

The same reasons as in $3 D$ systems lead to electron density contact perturbations in $2 D$ conducting structures. However, due to the peculiarities of low dimensional screening this perturbation falls off as $1 / x$ and hence has no special localization length. As a result, the use of metallic source-drain terminals, which is typical of $2 D$ transport measurements, leads to the perturbations of the electron density practically along the entire $2 D$ system. It is evident that this phenomen is very important for different transport problems in low dimensional electron systems, for example Quantum Hall Effect, different size effects, etc.

One promising way for the investigation of $2 D$ contact phenomena is based on the idea of depositing electrons on a liquid helium film condensed onto the surface of a $2 D$ sample in the presence of metallic terminals. The density of this classical 2DEG which adjusts to screen the potential from the sample provides a map. This charge density map may be read by optically measuring the variation in film thickness produced by the electrostatic pressure due to the charges, a technique aready employed for the investigation of multi-electron dimples on helium [3].

One favourable detail of the presented method is its realization under dc conditions. The existing alternative technique based on the linear electro-optic effect already used for the mapping of $2 D$ potential distributions [4-6] needs an ac perturbation. Besides the electrons on a helium film could realize the mapping without total screening of the $2 D$ electric potential distribution while the linear electro-optic effect is realized in the presence of an additional gate only.

In this paper we investigate the possible use of charged helium films for the mapping of model potential perturbations and demonstrate that this technique proves to be very promising for the applications outlined above.

A. Let us start from the solution of the electrostatic problem for a gated Corbino sample schematically shown in Fig. 1. 


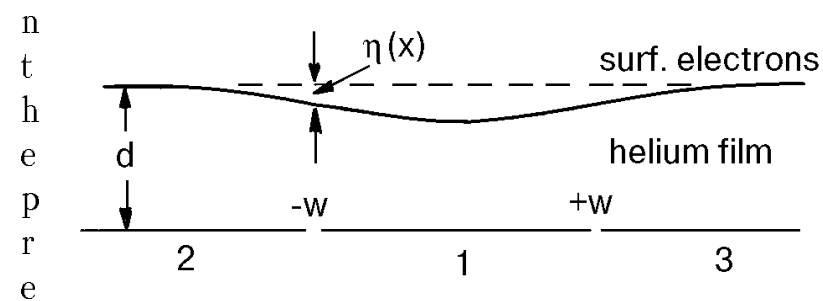

SFig. 1. Geometry and notations of the mapping problems with the electrons on helium film.

$\mathrm{e}$

nce of electro-potential difference $\Delta V$ between the stripe 1 and terminals 2,3 we have the following electron density distributions along the Corbino system for different screening levels (see Fig. 2, $a-c$ ). If Corbino is practically ungated (Fig. 2,a), the extra electrons are distributed mainly between the electrodes 2-1-3. The integral neutrality requirement is then fulfilled without taking the gate into account.

In the opposite limit (Fig. 2,c) the gate screening comes in, and the central Corbino part 1 has an essential, practically uniform fraction of extra elec-

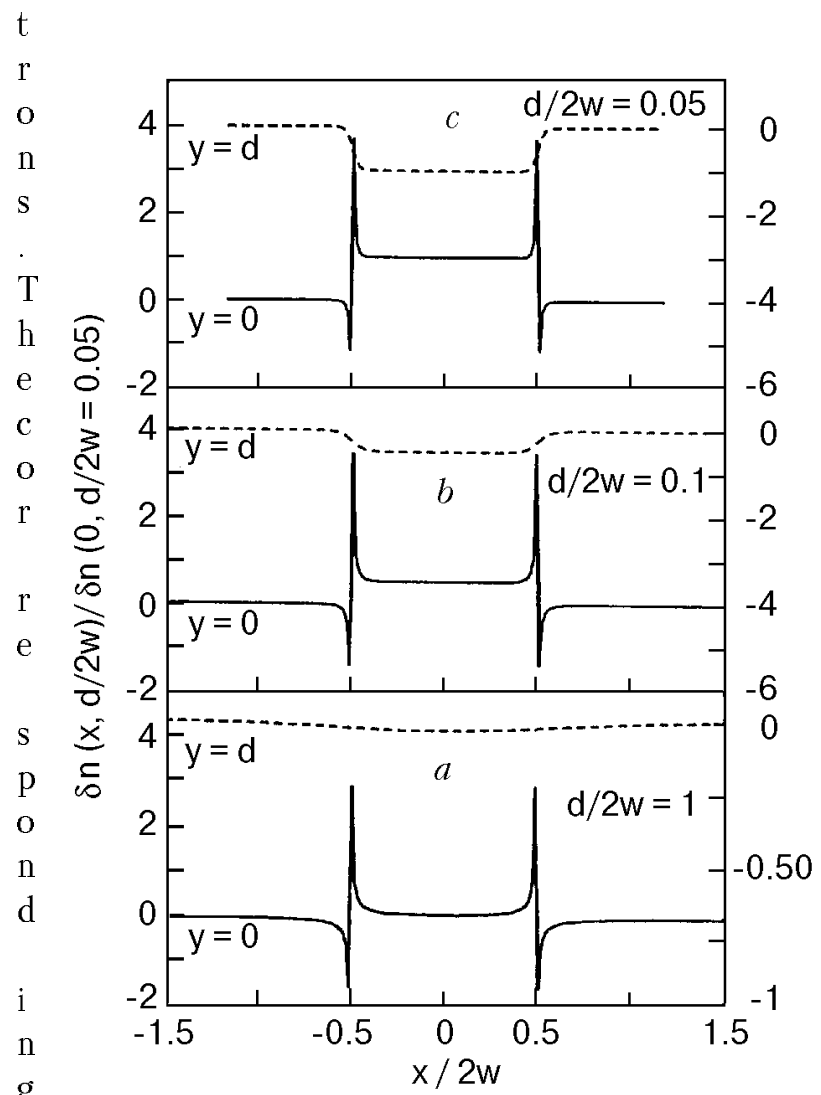

Fig. 2. Charge distribution along the gated Corbino disk with the potential perturbation between the electrodes $2-1-3$ for different screening levels $d / w$. Here $2 w$ is the width of the cen-

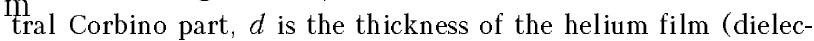
tric spacer). Solid lines correspond to Corbino sample. Dashed Pines show the gate charge distribution. All density distributions eare normalized to the distribution with the ratio $d / w=0.01$.

n sation charge is distributed mainly along the gate. This interesting peculiarity of $2 D$ contact phenomena is important for interpretation of many experimental results, e.g., the edge nature of minimal magnetocapacitance [7], unusual $S \mathrm{~d} H$ oscillations in a gated Corbino disk [8], etc. The same density distribution peculiarity is important for the potential mapping by electrons on helium film.

B. The point is that a non-uniform electron density distribution along the gate leads to a nonuniform deformation of the helium film, which could cover the Corbino sample (see Fig. 1) and this deformation can be detected optically using well-known methods [3,9]. Therefore the mapping of non-uniform potential perturbations along the $2 D$ electron system by electrons on the helium film is reduced to two steps. First, a charged helium film with a given $2 D$ system as a substrate is prepared, and second the optical technique [3,9] is applied to

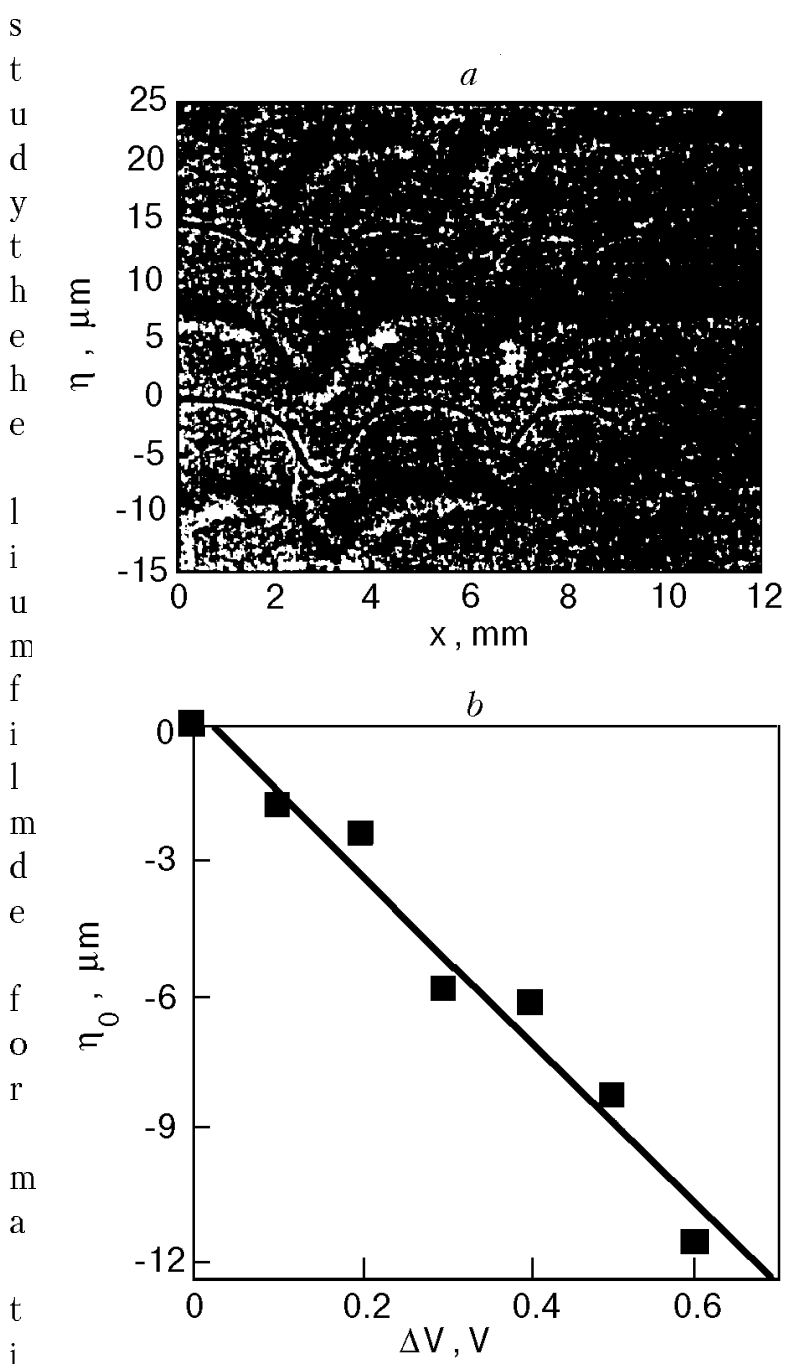

fig. 3. Calculations compared to the experimental image. Four perturbations in these figures correspond to different $w(1 ; 0.5$; 0.2 ; and $0.1 \mathrm{~mm}$ ) (a). Measured helium film deformation depth against the applied perturbation voltage $\Delta V$ for $2 w=1 \mathrm{~mm}$, $\mathrm{a}_{d}=100 \mu \mathrm{m}(b)$.

$\mathrm{u}$ 
sed by a non-uniform electron distribution along the helium film due to the screening redistribution of these electrons in the presence of potential perturbations in $2 D$ electron system. Realization of this program for the system shown in Fig. 1 is presented in Fig. 3 where Fig. 3, $a$ shows the comparison between the optical image $\eta(x)$ of the deformed helium film via $\Delta V \neq 0$ perturbation and corresponding calculations $\eta(x)$ using the solution of Poisson and mechanic equilibrium equations. Figure $3, b$ demonstrates a linear behaviour of the helium film deformation $\eta(0)$ versus applied voltage $\Delta V$. Solid line in this figure corresponds to the calculations $\eta(0)$ without adjustable parameters.

C. The above information leads to the conclusion that mapping of $2 D$ contact perturbations by electrons on a liquid helium film has good prospects. This method is suitable for a general presentation of the potential map. Besides, it can be useful to extract quantitative information about the details of low dimensional contact phenomena.

Some qualitative conclusions follow from the curves plotted in Fig. 2. These results show that in the absence of an additional gate a non-uniform electron density is developed along the entire $2 D$ electron system. In the presence of an additional gate, this perturbation is mainly uniform, but it still extends along the whole $2 D$ system.

D. This activity is partly supported by INTAS 93-939 and by NASA-PSA NAS 15-10110, project TM-17.

1. L. D. Landau and E. M. Lifshitz, Electrodynamics of Continous Media, Nauka, Moscow (1957) [in Russian].

2. S. M. Sze, Physics of Semiconductor Devices, John Wiley and Sons, New York, Chichester, Brisbane, Toronto, Singapore (1981).

3. P. Leiderer, W. Ebner, and V. Shikin, Surf. Sci. 113, 405 (1982).

4. P. F. Fontein, P. Hendriks, F. A. P. Blom, I. K. Wolter, L. I. Giling, and C. W. I. Beenaker, Surf. Sci. 963, 91 (1992).

5. R. Knott, W. Dietsche, K. v. Klitzing, K. Plong, and K. Eberle, Semic. Sci. Technol. 10, 117 (1995).

6. W. Dietsche, K. v. Klitzing, and U. Ploog, Surf. Sci. 361/362, 289 (1996).

7. S. Takaoka, K. Oto, H. Kurimoto, K. Murase, K. Gamo, and S. Nishi, Phys. Rev. Lett. 72, 3080 (1994).

8. V. T. Dolgopolov, A. A. Shashkin, G. V. Kravchenko et al., Pis'ma Zh. Eksp. Teor. Fiz. 63, 55 (1996).

9. D. Savignac and P. Leiderer, Phys. Rev. Lett. 49, 1869 (1982). 